\title{
NONNEGATIVE SOLUTIONS OF THE RADIAL LAPLACIAN WITH NONLINEARITY THAT CHANGES SIGN
}

\author{
N. P. CAC, A. M. FINK, AND J. A. GATICA
}

(Communicated by Hal L. Smith)

\begin{abstract}
We find a solution to the radial Laplacian equation $y^{\prime \prime}+\frac{N-1}{x} y^{\prime}+$ $\lambda a(x) f(y)=0, y^{\prime}(0)=y(1)=0$ when $a$ may change sign and is "sufficiently positive". The function $f$ is qualitatively like $e^{y}$, and we conclude solutions for $0 \leq \lambda \leq \lambda_{0}$.
\end{abstract}

\section{INTRODUCTION}

A variety of papers have looked at boundary value problems of the form

$$
\begin{aligned}
& L y=\lambda a(x) f(y), \\
& y(0)=y(1)=0,
\end{aligned}
$$

or

$$
y^{\prime}(0)=y(1)=0
$$

in which $f \geq 0$ and $a \geq 0$, and $L$ is some differential operator. We have discussed this problem in [1], [2], [3], and [4] as well as other papers cited there. In each case we have endeavored to show the existence of solutions by constructive means, that is, have some sort of iteration converge. If $f(0)=0$ it is especially difficult to get a numerical procedure to converge to a positive solution. In this paper, we continue this study in which we allow, for the first time, the coefficient function $a$ to change sign.

To be specific we study the problem $(1)$ and $\left(2^{\prime}\right)$ where $L y=-\left[y^{\prime \prime}+\frac{N-1}{x} y^{\prime}\right]$ for $N \geq 2$. Obvious modifications in our arguments would also suffice for the problem (1) and (2) with $L y=-y^{\prime \prime}$.

We are considering finding a positive solution $(y>0$ in $[0,1))$ of

$$
y^{\prime \prime}+\frac{N-1}{x} y^{\prime}+\lambda a(x) f(y)=0
$$

with

$$
y^{\prime}(0)=y(1)=0
$$

Received by the editors June 18, 1993.

1991 Mathematics Subject Classification. Primary 34B15, $35 \mathrm{~J} 67$.

Key words and phrases. Elliptic boundary value problem, radially symmetric solution, Green's function. 
where $\lambda$ is a positive parameter;

$$
a(t)=a_{+}(t)-a_{-}(t) \quad \text { with } a_{ \pm}(t) \geq 0 \text { and } a \in L_{1}(0,1)
$$

and

$f$ is continuous, positive, and nondecreasing on $[0, \infty)$.

It is well known that the Green's function $G(x, t)$ for (3) and (4) is nonnegative when we write it as

$$
\left(x^{N-1} y^{\prime}\right)^{\prime}+\lambda x^{N-1} a(x) y(x)=0 .
$$

Moreover it is easy to see that a solution to (3) and (4) is a fixed point of the operator

$$
T \Phi(x) \equiv \lambda \int_{0}^{1} G(x, t) t^{N-1} a(t) f(\Phi(t)) d t
$$

whose domain will be $C^{+}(0,1)$, the cone of continuous non-negative functions. The operator $T$ does not leave this cone invariant since we allow $a(x)$ to change sign.

In order to set up a convergent iteration we will use the following proposition.

Proposition. Assume (5) and (6). Let $A=\{x \mid a(x) \geq 0\}$ and $B=\{x \mid a(x)<$ $0\}$. Suppose we have bounded measurable functions $\phi_{0}, \psi_{0}$ on $[0,1]$ such that they satisfy

$$
0 \leq \phi \leq \psi \quad \text { on } A, \quad 0 \leq \psi \leq \phi \quad \text { on } B
$$

$$
T \psi \leq \psi \quad \text { on } A, \quad T \psi \leq \phi \quad \text { on } B
$$

and

$$
T \phi \geq \phi \quad \text { on } A, \quad T \phi \geq \psi \text { on } B
$$

\section{Define}

$$
\phi_{1}(x)=\left\{\begin{array}{ll}
T \phi_{0}(x) & \text { on } A, \\
T \psi_{0}(x) & \text { on } B ;
\end{array} \quad \psi_{1}(x)= \begin{cases}T \psi_{0}(x) & \text { on } A, \\
T \phi_{0}(x) & \text { on } B\end{cases}\right.
$$

Then $\phi_{1}$ and $\psi_{1}$ also satisfy (i), (ii), and (iii).

Proof. We note that the operator $T$ can be written as

$$
\begin{aligned}
T \phi(x)= & \lambda \int_{A} G(x, t) t^{N-1} a_{+}(t) f(\phi(t)) d t \\
& -\lambda \int_{B} G(x, t) t^{N-1} a_{-}(t) f(\phi(t)) d t .
\end{aligned}
$$

For convenience we write

$$
T \phi(x)=T_{1} \phi(x)-T_{2} \phi(x)
$$

where $T_{i} \phi, i=1,2$, are both monotone in the sense that $\phi \leq \psi$ implies $T_{i} \phi \leq T_{i} \psi . T_{1}$ acts on $C^{+}([0,1] \cap A)$ and $T_{2}$ on $C^{+}([0,1] \cap B)$. Note that (i) implies that

$$
T \phi_{0}=T_{1} \phi_{0}-T_{2} \phi_{0} \leq T_{1} \psi_{0}-T_{2} \psi_{0}=T \psi_{0}
$$


This immediately implies that $\phi_{1}$ and $\psi_{1}$ satisfy (i). The condition (ii) for $\psi_{1}$ is that on $A, T \psi_{1} \leq \psi_{1}=T \psi_{0}$ and on $B, T \psi_{1} \leq \phi_{1}=T \psi_{0}$, i.e., $T \psi_{1} \leq T \psi_{0}$. But using (ii) and (iii)

$$
T \psi_{1}=T_{1}\left(T \psi_{0}\right)-T_{2}\left(T \phi_{0}\right) \leq T_{1}\left(\psi_{0}\right)-T_{2}\left(\psi_{0}\right)=T \psi_{0} .
$$

Similarly condition (iii) for $\phi_{1}$ is that $T \phi_{1} \geq \phi_{1}=T \phi_{0}$ on $A$ and $T \phi_{1} \geq$ $\psi_{1}=T \psi_{0}$ on $B$. Now

$$
T \phi_{1}=T_{1}\left(T \phi_{0}\right)-T_{2}\left(T \psi_{0}\right) \geq T_{1}\left(\phi_{0}\right)-T_{2}\left(\phi_{0}\right)=T \phi_{0}
$$

gives (iii) for $\phi_{1}$.

Theorem 1. Assume (5) and (6), and suppose there are functions $\phi_{0}, \psi_{0}$ that satisfy (i), (ii), and (iii). Then the problem (3)-(4) has a solution.

Proof. We define

$$
\phi_{n+1}=\left\{\begin{array}{ll}
T \phi_{n} & \text { on } A, \\
T \psi_{n} & \text { on } B ;
\end{array} \quad \psi_{n+1}= \begin{cases}T \psi_{n} & \text { on } A, \\
T \phi_{n} & \text { on } B .\end{cases}\right.
$$

By the proposition and induction, $\left(\phi_{n}, \psi_{n}\right)$ satisfies (i), (ii), and (iii) and hence (10), (11), and (12), i.e.,

$$
0 \leq T \phi_{n} \leq T \phi_{n+1} \leq T \psi_{n+1} \leq T \psi_{n} \leq T \psi_{0} .
$$

Thus $T \phi_{n} \uparrow \bar{\phi}$ and $T \psi_{n} \downarrow \bar{\psi}$ pointwise and $\bar{\phi} \leq \bar{\psi}$. Since

$$
T \phi_{n+1}=T_{1}\left(T \phi_{n}\right)-T_{2}\left(T \psi_{n}\right),
$$

we may apply Lebesgue's dominated convergence theorem to have

$$
\bar{\phi}=T_{1}(\bar{\phi})-T_{2}(\bar{\psi})
$$

so that $\bar{\phi}$ is continuous on $[0,1]$. Similarly $\bar{\psi}$ is continuous on $[0,1]$, and

$$
\bar{\psi}=T_{1}(\bar{\psi})-T_{2}(\bar{\phi}) \text {. }
$$

From (13) we have $\phi_{n+1} \rightarrow \bar{\phi}$ on $A$ by definition of $\bar{\phi}$. On the other hand $\phi_{n+1}=T \phi_{n}$ on $A$, so $\phi_{n+1} \rightarrow T \bar{\phi}$. Thus $\bar{\phi}=T \bar{\phi}$ on $A$. On $B$ we have $\psi_{n+1}=T \phi_{n} \rightarrow \bar{\phi}$ by definition of $\bar{\phi}$. But $\phi_{n+1}=T \psi_{n}$ on $B$, so $\phi_{n+1} \rightarrow \bar{\psi}$ on $B$. Thus on $B, T \bar{\psi}=\bar{\phi}$. In a similar way we have

$$
\bar{\phi}=\left\{\begin{array}{ll}
T \bar{\phi} & \text { on } A, \\
T \bar{\psi} & \text { on } B ;
\end{array} \quad \bar{\psi}= \begin{cases}T \bar{\psi} & \text { on } A, \\
T \bar{\phi} & \text { on } B,\end{cases}\right.
$$

and $\bar{\phi}$ and $\bar{\psi}$ are fixed points of $T^{2}$.

Now consider the convex region in $C[0,1]$ defined by $C=\{g(x) \mid \bar{\phi}(x) \leq$ $g(x) \leq \bar{\psi}(x), x \in[0,1]\}$. This is invariant under $T$ for

$$
T g=T_{1}(g)-T_{2}(g) \leq T_{1}(\bar{\psi})-T_{2}(\bar{\phi})=\bar{\psi}
$$

by (15). Similarly

$$
T g=T_{1}(g)-T_{2}(g) \geq T_{1}(\bar{\phi})-T_{2}(\bar{\psi})=\bar{\phi} .
$$

Now $G(x, t) t^{N-1}$ is continuous on $[0,1] \times[0,1]$, so $a \in L_{1}(0,1)$ implies that $\{(T g) \mid g \in C\}$ is uniformly equicontinuous, so $T$ restricted to $C$ is a compact operator. By Schauder's fixed point theorem $T$ has a fixed point. 
Comment. The order interval $\left\{g(x) \mid T \phi_{0} \leq g \leq T \psi_{0}\right\}$ already is invariant and one could use Schauder's theorem directly. But the iteration improves the estimates. In general one might expect the two functions $\bar{\phi}$ and $\bar{\psi}$ to be the same. So one could in effect construct the solution numerically.

Observation. If $f \geq 0$ and nonincreasing, then one takes (i) and the following modified versions:

$$
\begin{aligned}
& T \phi \leq \psi \quad \text { on } A, \quad T \phi \leq \phi \quad \text { on } B \text {; } \\
& T \psi \geq \phi \quad \text { on } A, \quad T \psi \geq \psi \quad \text { on } B \text {; }
\end{aligned}
$$

and

$$
\phi_{1}=\left\{\begin{array}{ll}
T \psi_{0} & \text { on } A, \\
T \phi_{0} & \text { on } B ;
\end{array} \quad \psi_{1}= \begin{cases}T \phi_{0} & \text { on } A, \\
T \psi_{0} & \text { on } B .\end{cases}\right.
$$

One can prove the theorem of existence where now $T \phi \geq T \psi, T \phi \downarrow$, and $T \psi \uparrow$. The details are the same.

\section{AN EXAMPLE}

To construct an example where the previous analysis applies, we will make the following assumption.

$(\mathrm{H})$ : There is an $\varepsilon>0$ so that

$$
\int_{0}^{t} x^{N-1} a_{+}(x) d x \geq(1+\varepsilon) \int_{0}^{t} x^{N-1} a_{-}(x) d x \quad \text { for all } t \in[0,1] .
$$

Comment. $H$ means $a$ is sufficiently positive near 0 .

We seek $\phi_{0}$ and $\psi_{0}$ so that (i), (ii), and (iii) are satisfied. Let $\phi_{0}(x)=\alpha$ on $B$ and $\phi_{0}(x)=0$ on $A$ with $\psi_{0}(x)=\alpha$ on $A$ and $\psi_{0}(x)=0$ on $B$. Then (i) is satisfied if $\alpha \geq 0$. Now the condition (ii) is

$$
T \psi_{0}=T_{1}(\alpha)-T_{2}(0) \leq \alpha \text { on }[0,1]
$$

while (iii) is

$$
T \phi_{0}=T_{1}(0)-T_{2}(\alpha) \geq 0 \text { on }[0,1] .
$$

Letting $z_{ \pm}(x) \equiv \int_{0}^{1} G(x, t) t^{N-1} a_{ \pm}(t) d t$ these conditions become

$$
\lambda\left[z_{+}(x) f(\alpha)-z_{-}(x) f(0)\right] \leq \alpha
$$

and

$$
\lambda\left[z_{+}(x) f(0)-z_{-}(x) f(\alpha)\right] \geq 0 .
$$

We consider $(18)$ first. Define $z(x)=z_{+}(x)-(1+\varepsilon) z_{-}(x)$. Then $z$ is a solution of

$$
z^{\prime \prime}+\frac{N-1}{x} z+\left(a_{+}-(1+\varepsilon) a_{-}\right)=0, \quad z^{\prime}(0)=z(1)=0 .
$$

It follows that

$$
\left(z^{\prime} x^{N-1}\right)^{\prime}=-x^{N-1}\left(a_{+}(x)-(1+\varepsilon) a_{-}(x)\right)
$$

and

$$
z^{\prime}(t) t^{N-1}=-\int_{0}^{t} x^{N-1}\left(a_{+}(x)-(1+\varepsilon) a_{-}(x)\right] d x \leq 0
$$


by $(\mathrm{H})$. Thus $z$ is decreasing and therefore is non-negative, i.e., $z_{+}(x) \geq$ $(1+\varepsilon) z_{-}(x)$ on $[0,1]$. So $(18)$ is satisfied if

$$
f(\alpha) \leq(1+\varepsilon) f(0) .
$$

We select such an $\alpha$ and argue that (17) can now be satisfied for small $\lambda$. To prove this we will give an explicit estimate. Now as above

$$
z_{+}(x)-z_{-}(x)=\int_{0}^{1} G(x, t) t^{N-1} a(t) d t
$$

and the right-hand side is a decreasing function, so

$$
z_{+}(x) \leq z_{-}(x)+\int_{0}^{1} G(0, t) t^{N-1} a(t) d t .
$$

This last integral is

$$
\frac{1}{N-2} \int_{0}^{1} t\left(1-t^{N-2}\right) a(t) d t \equiv \beta \quad\left(\int_{0}^{1} \ln t a(t) d t \equiv \beta \text { if } N=2\right) .
$$

Hence

$$
\begin{aligned}
f(\alpha) z_{+}(x)-f(0) z_{-}(x) & \leq[f(\alpha)-f(0)] z_{-}(x)+f(\alpha) \beta \\
& \leq[f(\alpha)-f(0)] z_{-}(0)+f(\alpha) \beta
\end{aligned}
$$

since $z_{-}$is decreasing (as above for $z$ ).

This in turn is dominated by (see (19))

$$
\varepsilon f(0) z_{-}(0)+(1+\varepsilon) f(0) \beta .
$$

So (17) is satisfied if

$$
\lambda \leq \frac{\alpha}{f(0)\left[\varepsilon z_{-}(0)+(1+\varepsilon) \beta\right]}
$$

the denominator is explicitly $(N \geq 3)$

$$
\begin{aligned}
f(0) & {\left[\varepsilon \int_{0}^{1} \frac{\left(t-t^{N-1}\right)}{N-2} a_{-}(t) d t+(1+\varepsilon) \int_{0}^{1} \frac{\left(t-t^{N-1}\right)}{N-2} a(t) d t\right] } \\
& =f(0)\left[\int_{0}^{1} \frac{\left(t-t^{N-1}\right)}{N-2} a(t) d t+\varepsilon \int_{0}^{1} \frac{\left(t-t^{N-1}\right)}{N-2} a_{+}(t) d t\right] .
\end{aligned}
$$

Theorem. If $f(0)>0, f$ is non-decreasing, and $a$ is measurable and in $L^{1}(0,1)$ such that $(\mathrm{H})$ is satisfied, then problem $(1)-\left(2^{\prime}\right)$ has a solution for $0 \leq \lambda \leq \lambda_{0}$ where $(N \geq 3)$

$$
\lambda_{0}=\frac{(N-2) \alpha}{f(0) \int_{0}^{1}\left(t-t^{N-1}\right) a(t) d t+\varepsilon \int_{0}^{1}\left(t-t^{N-1}\right) a_{+}(t) d t}
$$

if $f(\alpha) \leq f(0)(1+\varepsilon)$. For $N=2$,

$$
\lambda_{0}=\frac{-\alpha}{f(0) \int_{0}^{1} t \ln t a(t) d t+\varepsilon \int_{0}^{1} t \ln t a_{+}(t) d t} .
$$


For specific examples, one can take $f(\alpha)=f(0)(1+\varepsilon)$. For example, if $f(y)=e^{y}, \alpha=\ln (1+\varepsilon)$, and $f(y)=1+y^{P}$, then $\alpha=\varepsilon^{1 / p}$. For an example of $(\mathrm{H})$ take

$$
a(t)= \begin{cases}A, & 0 \leq t<\frac{1}{2}, \\ -B, & \frac{1}{2}<t \leq 1,\end{cases}
$$

where $0<B\left(2^{N}-1\right)<A$. One can easily verify that $1+\varepsilon=\frac{A}{B\left(2^{N}-1\right)}$ will work for $(\mathrm{H})$. In fact if $a(t)$ is decreasing on $[0,1]$ with $a\left(x_{0}\right)=0$, then $\int_{0}^{t} x^{N-1} a_{+}(x) d t \geq 0$ on $\left[0, x_{0}\right]$ and equal to $\int_{0}^{x_{0}} x^{N-1} a_{+}(x) d x$ on $\left[x_{0}, 1\right]$. Since $\int_{0}^{t} x^{N-1} a_{-}(x) d x=0$ on $\left[0, x_{0}\right],(\mathrm{H})$ is satisfied if $\int_{0}^{x_{0}} x^{N-1} a_{+}(x) d x \geq$ $(1+\varepsilon) \int_{0}^{t} x^{N-1} a_{-}(x) d x$ on $\left[x_{0}, 1\right]$. Since the right-hand side is increasing, the condition is

$$
(1+\varepsilon) \leq \frac{\int_{0}^{1} x^{N-1} a_{+}(x) d x}{\int_{0}^{1} x^{N-1} a_{-}(x) d x} .
$$

We have given a sufficient condition for small eigenvalues of the problem (1) $-\left(2^{\prime}\right)$ which involve $a_{+}$being sufficiently positive, i.e., the condition $(\mathbf{H})$. Some such hypothesis is necessary, but we believe that $\lambda$ small is a correct condition.

\section{REFERENCES}

1. A. M. Fink, J. A. Gatica, G. E. Hernandez, and P. Waltman, Approximation of solutions of singular second order boundary value problems, SIAM J. Math. Anal. 22 (1991), 440-462.

2. A. M. Fink, The radial Laplacian Gelfand problem, Proc. Diff. and Delay Equations Conference (A. M. Fink, W. Kliemann, and R. K. Miller, eds.), World Scientific Publications, Singapore, 1992, pp. 93-98.

3. A. M. Fink, J. A. Gatica, and G. E. Hernandez, Eigenvalues of generalized Gelfand models, J. Nonlinear Anal. 20 (1993), 1453-1468.

4. N. P. Cac, A. M. Fink, and J. A. Gatica, Nonnegative solutions of quasilinear elliptic problems with nonnegative coefficients, preprint.

(N. P. Cac and J. A. Gatica) Department of Mathematics, University of Iowa, Iowa City, IowA 52242

E-mail address, N. P. Cac: nguyen-cac@uiowa.edu

E-mail address, J. A. Gatica: jgatica@blue.weeg.uiowa.edu

(A. M. Fink) Department of Mathematics, Iowa State University, Ames, Iowa 50011

E-mail address: fink@pollux.math.iastate.edu 